\title{
Guaranteeing motion safety for robots
}

\author{
Thierry Fraichard • James J. Kuffner Jr.
}

Received: 18 January 2012 / Accepted: 25 January 2012 / Published online: 7 February 2012

(C) Springer Science+Business Media, LLC 2012

\section{Introduction}

In the near future, it is expected that robotic systems will share the human living and working spaces: service robots and cybernetic transport systems are examples of two primary application areas. The technology is now mature; we have already witnessed autonomous mobile robots guiding people in museums and automated cars driving on the road network.

While moving (especially at high speed), automated vehicles, mobile manipulators and humanoid robots can be potentially dangerous should a collision occur. Before letting such robotic systems transport or share space with people in a truly autonomous way, it is critical to assert and characterize their motion safety, i.e. their ability to avoid collision. Demonstrating that a robotic system is working properly on a limited set of experiments is not enough. If autonomous robots are ever to be deployed among human beings on a large scale, there is a need to characterize the level of motion safety that can be achieved and/or to specify the conditions under which it can be guaranteed. Collisions happen for reasons that broadly fall into one of the following category:

- Hardware failures, e.g. brake failure.

- Software bugs, e.g. truncation error.

- Perceptual errors, i.e. all the errors that are related to the sensing system of the robot and that result in the robot

T. Fraichard $(\bowtie)$

INRIA Grenoble Rhône-Alpes, 655 avenue de l'Europe,

38330 Montbonnot, France

e-mail: thierry.fraichard@inria.fr

J.J. Kuffner Jr.

The Robotics Institute, Carnegie Mellon University, Pittsburgh,

PA, USA

e-mail: kuffner@cs.cmu.edu having an incorrect understanding of its environment (e.g. false negative).

- Reasoning errors, i.e. at a certain point a wrong decision was made.

The purpose of this special issue of Autonomous Robots is to explore the motion safety issue from the decision-making point of view (fourth item of the list above) assuming that the robotic system at hand is working alright (from the hardware and software point of view) and has an accurate understanding of its current situation (no perceptual errors).

Roboticists have long been aware of the motion safety issue. There is a rich literature on collision avoidance starting with the pioneering work of Moravec (1981). The motion safety issue was recently explored at an abstract level by Fraichard (2007). If needed be, it highlighted a number of requirements whose violation is likely to put a robotic system into danger and yield collisions. These requirements are fairly intuitive and straightforward to express. In static environments, the key thing is to take into account the dynamic properties of the robotic system at hand, e.g. its momentum, its steering capabilities. In dynamic environments, things become more complicated: it becomes necessary to reason about the future evolution of the environment and do so with an appropriate lookahead, i.e. how far into the future the reasoning is done. In other words, it requires a spacetime model of the future which is in itself a challenge to build inasmuch as, in most real-world situations, complete information about the environment and its future evolution is not available (not to mention the fact that the actions of the robot at hand influence the environment's future evolution). Because of all these challenging issues, guaranteed motion safety in the real world remains an open problem.

The nine papers comprising this special issue address different collision avoidance instances of varying complexity: one robot $v s$ multiple robots; $2 \mathrm{D}$ vs $3 \mathrm{D}$ environments, static 
vs dynamic environments, etc. The tools and the techniques employed are diverse as well, e.g. Lyapunov theory, Coordination Diagrams (O'Donnell and Lozano-Perez 1989), Velocity Obstacles (Fiorini and Shiller 1998)/Collision Cones (Chakravarthy and Ghose 1998), Inevitable Collision States (Fraichard and Asama 2004). But, in all cases, the authors address motion safety in a formal way and spell out both the conditions under which their approach apply and the level of motion safety that is achieved.

\section{Guide to the special issue}

The first two papers address motion safety in 2D static environments. The first paper by Lapierre and Zapata considers a single robot case. The authors propose a reactive path following control technique for a nonholonomic unicycle. Their solution is based on recent advances in nonlinear control theory. It guarantees asymptotic convergence to the path and obstacle avoidance. The second paper by Cui et al. considers a multiple robots case. The authors present a centralised approach to coordinate in a Pareto-optimal way the motions of a set of mobile robots. Their solution uses path/velocity decomposition (Kant and Zucker 1986) and Coordination Diagrams.

The next three papers consider dynamic environments and are all based on the Velocity Obstacle/Collision Cone concept. The first paper by Lalish and Morgansen considers a multiple robots case. Unlike the previous paper, the authors propose a distributed reactive collision avoidance approach. It is primarily based on a Collision Cone-based feedback control law. The second paper by $\mathrm{Wu}$ and How considers a single robot case. Its main contribution is an extension of the Velocity Obstacle concept that uses reachable sets for the future motions of the moving obstacles instead of single predicted trajectories. The third paper by Chakravarthy and Ghose seeks to extend the Collision Cone Concept that has been so fruitful in 2D to $3 \mathrm{D}$ environments.

The next two papers also consider dynamic environments, unlike the three previous ones, they are based on the Inevitable Collision State concept. The first paper by Bouraine et al. considers the case of a single robot with a limited field-of-view. Because collision avoidance is impossible to guarantee in such a situation, the authors settle for a weaker level of motion safety based on Braking Inevitable Collision States. The second paper by Althoff et al. is based on a probabilistic version of the Inevitable Collision States (Bautin et al. 2010). Because of this choice, the paper is about safety assessment rather than strict collision avoidance per se.

The last two papers again considers static environments. Although the collision avoidance problem addressed in the first paper by Taübig et al. may appear limited (2D static obstacle avoidance), the authors show what it takes for a software component to be certified for industry applications using formal software verification tools.

Finally, while all the previous papers considered singlebody robots, the last paper by Rubrecht et al. deals with a 6 degrees-of-freedom robot arm, i.e. a multi-body robot, moving in a 3D static environment. In a teleoperation context, the authors present a methodology to ensure safety at the control level.

\section{Papers in the special issue}

1. Lapierre L, Zapata R (2012) A guaranteed obstacle avoidance guidance system. Autonomous Robots. doi: 10.1007/s10514-011-9269-5.

2. Cui R, Gao B, Guo J (2012) Pareto-optimal coordination of multiple robots with safety guarantees. Autonomous Robots. doi:10.1007/s10514-011-9265-9.

3. Lalish E, Morgansen K (2012) Distributed reactive collision avoidance. Autonomous Robots. doi:10.1007/ s10514-011-9267-7.

4. Wu A, How J (2012) Guaranteed infinite horizon collision avoidance of unpredictable, dynamically constrained obstacles. Autonomous Robots. doi:10.1007/ s10514-011-9266-8.

5. Chakravarthy A, Ghose D (2012) Generalization of the collision cone approach for motion safety in 3-d environments. Autonomous Robots. doi:10.1007/s10514-0119270-z.

6. Bouraine S, Fraichard T, Salhi H (2012) Provably safe navigation for mobile robots with limited field-of-views in dynamic environments. Autonomous Robots. doi: 10.1007/s10514-011-9258-8.

7. Althoff D, Kuffner J, Wollherr D, Buss M (2012) Safety assessment of robot trajectories for navigation in uncertain and dynamic environments. Autonomous Robots. doi:10.1007/s10514-011-9257-9.

8. Taübig H, Frese U, Hertzberg C, Luth C, Mohr S, Vorobev E, Walter D (2012) Guaranteeing functional safety: design for provability and computer-aided verification. Autonomous Robots. doi:10.1007/s10514-0119271-y.

9. Rubrecht S, Padois V, Bidaud P, de Broissia M, da Silva Simoes M (2012) Motion safety and constraints compatibility for multibody robots. Autonomous Robots. doi: 10.1007/s10514-011-9264-X.

Acknowledgements The guest editors wish to express their sincere gratitude to Gaurav Sukhatme, Editor-in-Chief of Autonomous Robots, for giving them the opportunity to edit this special issue, for his competent guidance and for being flexible about the deadlines. They also thank Rolan Profugo, Suganthi Duraisingam and Tim Stout from Springer's Journal Editorial Office for their invaluable help throughout the whole editing process. Finally, they are deeply indebted to the many colleagues who provided thorough and helpful reviews for the papers submitted to the Special Issue. 


\section{References}

Bautin, A., Martinez-Gomez, L., \& Fraichard, T. (2010). Inevitable collision states: a probabilistic perspective. In IEEE int. conf. on robotics and automation, Anchorage, US.

Chakravarthy, A., \& Ghose, D. (1998). Obstacle avoidance in a dynamic environment: A collision cone approach. IEEE Transactions on Systems, Man, and Cybernetics, Part A, 28(5).

Fiorini, P., \& Shiller, Z. (1998). Motion planning in dynamic environments using velocity obstacles. International Journal of Robotics Research, 17(7).

Fraichard, T. (2007). A short paper about motion safety. In IEEE int. conf. on robotics and automation, Roma, IT.

Fraichard, T., \& Asama, H. (2004). Inevitable collision states. a step towards safer robots? Advanced Robotics, 18(10).

Kant, K., \& Zucker, S. (1986). Toward efficient trajectory planning: The path-velocity decomposition. International Journal of Robotics Research, 5(3).

Moravec, H. (1981). Rover visual obstacle avoidance. In Int. joint conf. on artificial intelligence, Vancouver, CA.

O’Donnell, P., \& Lozano-Perez, T. (1989). Deadlock-free and collision-free coordination of two robot manipulators. In IEEE int. conf. on robotics and automation, Scottsdale, US.

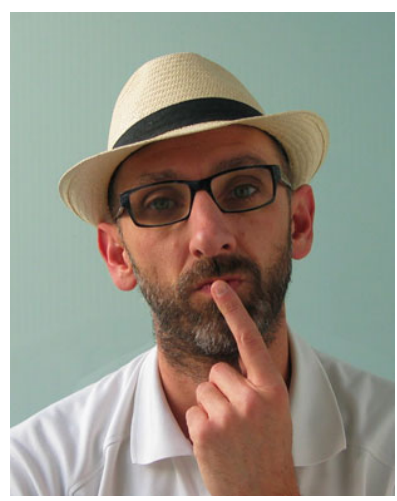

Thierry Fraichard is an INRIA Research Associate at the INRIA Grenoble Rhône-Alpes Research Center in France. He received a $\mathrm{Ph} . \mathrm{D}$. in Computer Science from the Institut National Polytechnique de Grenoble (INPG) in April 1992. From December 1993 to November 1994, he was a Postdoctoral Fellow in the Robotics Institute at Carnegie Mellon University in Pittsburgh, US. From November 2000 to January 2001, then again in November 2001, he was a Tan Chin Tuan Fellow at Nanyang Technological
University in Singapore. From September to December 2002, he was a Japan Society for the Promotion of Science (JSPS) Research Fellow at Riken Institute in Tokyo. From September 2007 to August 2008, he was a Visiting Professor at the Swiss Federal Institute of Technology in Zürich. His research focuses on motion autonomy for vehicles and mobile robots with a special emphasis on motion safety, motion planning, prediction of the future motion of moving objects, navigation amidst human beings, and the design of control architectures for autonomous vehicles.

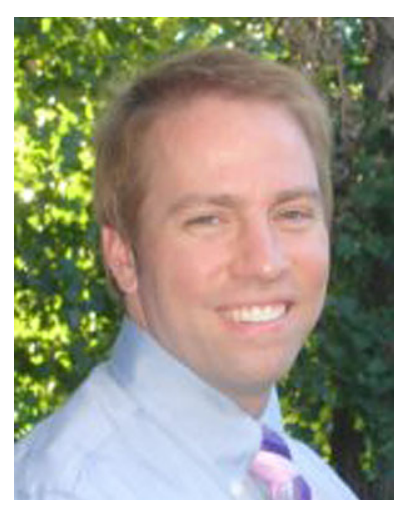

James J. Kuffner Jr. is an Adjunct Associate Professor at the Robotics Institute, Carnegie Mellon University currently working as a Research Software Engineer at Google, Inc. He received a Ph.D. from the Stanford University Dept. of Computer Science Robotics Laboratory in 1999. He spent two years as a Japan Society for the Promotion of Science (JSPS) Postdoctoral Research Fellow at the University of Tokyo working on software and planning algorithms for humanoid robots. He joined the faculty at Carnegie Mellon University's Robotics Institute in 2002. He has published over 100 technical papers and received the Okawa Foundation Award for Young Researchers in 2007. 